\title{
Prophylactic anticoagulant therapy decreases the incidence of deep vein thrombosis in patients with solid tumors: A systematic review and meta-analysis
}

\author{
Yunjiao Zhou ${ }^{1}$, Gong Yang*1, 2,3 ${ }^{\text {, Chenglei Huang }}{ }^{4}$ \\ ${ }^{1}$ Central Laboratory, The Fifth People's Hospital of Shanghai, Fudan University, Shanghai, China \\ ${ }^{2}$ Cancer Institute, Fudan University Shanghai Cancer Center, Shanghai, China \\ ${ }^{3}$ Department of Oncology, Fudan University Shanghai Medical College, Shanghai, China \\ ${ }^{4}$ Sino-American Shanghai Squibb Pharmaceuticals Ltd., Shanghai, China
}

Received: November 23, 2016

Accepted: December 16, 2016

Online Published: January 3, 2017

DOI: $10.5430 /$ jst.v7n1p35

URL: http://dx.doi.org/10.5430/jst.v7n1p35

\begin{abstract}
It is not well understood the efficacy and safety of primary deep vein thrombosis (DVT) prophylaxis of anticoagulants in patients with solid tumors. This systematic review and meta-analysis of randomized controlled trials (RCT) determines the relative ratio of primary DVT, survival rate and bleeding events among patients with solid tumors treated with anticoagulants or placebo. Comprehensive literature searches were conducted through the Pubmed, Ovid MEDLINE and EMBASE databases published from January 1st, 1993 to December 31st, 2015. Statistical analysis was performed by RevMan 5.0 software. For DVT events, the risk ratio in 16 trials between the prophylactic and control patients was statistically significant at 0.45 [0.36-0.58]; for major bleeding events, the risk ratio in 18 trials between the prophylactic and control patients was not statistically significant at 1.33 [0.99-1.79], while that in 15 trials with clinically relevant non-major bleeding was statistically significant at 1.83 [1.46-2.30]; the risk ratio for the mortality rate of patients with solid tumors in 16 trials was not statistically significant at 0.97 [0.93-1.02]. In conclusion, the risk ratio in this meta-analysis showed a significantly reduced incidence of DVT with anticoagulant use. Treatment to patients who had solid tumors with prophylactic anticoagulants enhanced the incidence rate of non-major bleeding but has no significant impact on the incidence rate of major bleeding. No significant differences were found in the mortality outcomes between anticoagulant and non-anticoagulant groups.
\end{abstract}

Key Words: Prophylactic anticoagulant, Deep vein thrombosis, Mortality, Solid tumors

\section{INTRODUCTION}

The risk of postoperative venous thromboembolism, including deep vein thrombosis (DVT) and pulmonary embolism $(\mathrm{PE}),{ }^{[1]}$ is reported to be twice higher in patients with cancer than in those without cancer. ${ }^{[2]}$ Thenceforth, several prospective or cohort studies demonstrated that compared with patients without cancer, patients with cancer, especially those with solid tumors had an increased risk of DVT. ${ }^{[3,4]}$ The incidence of thromboembolic events in patients with cancers varied from $1.6 \%$ to 5.3\%. ${ }^{[4-10]}$ Among thromboembolic patients, those with pancreatic cancer or brain cancer had the highest risk of DVT, 4- or 3-folds more than patients

\footnotetext{
*Correspondence: Gong Yang; Email: yanggong@fudan.edu.cn; Address: Central Laboratory, The Fifth People's Hospital of Shanghai, Fudan University, 200240, Shanghai, China.
} 
without cancers. ${ }^{[8,10]}$

The close association between hypercoagulability and risk of thrombosis in cancer patients has been recognized by $\mathrm{Ar}$ mand Trousseau since 1865. ${ }^{[11,12]}$ From then on, increasing studies suggested that the mechanism could be a positive feedback loop between tumor cells and haemostatic system. ${ }^{[13]}$ Besides this, chemotherapy was increasingly recognized as a risk factor for thromboembolic complications due to the induced damage of vascular endothelium, ${ }^{[14]}$ which may amplify the prothrombotic effects of cancer cells ${ }^{[5,15]}$ and damage vessel walls, ${ }^{[16,17]}$ leading to the activation of haemostatic system. So DVT is a leading cause of death among patients treated with chemotherapy, only second to progression of malignancy. ${ }^{[18]}$

The type of malignancy, tumor stage, surgeries, genetics, or even ages, sex and races also contribute to high risk of thromboembolic events in cancer patients. ${ }^{[19]}$ It is reported that 1-year survival rate of cancer patients with DVT is significantly shortened, only a third of those are without thrombosis. ${ }^{[20]}$ Once patients suffered from DVT, the treatment is highly intensive and costly. In a retrospective analysis, the mean length of hospitalization in cancer patients with DVT was 11 days, and the average cost of hospitalization was $\$ 20,065 .^{[21]}$

Since cancer patients may be at high risk of developing DVT and other types of thromboembolic events, ${ }^{[3,22]}$ DVT is recognized as a risk factor to predict mortality of cancer patients. However, routine antithrombotic prophylaxis is only recommended for cancer patients undergone surgery or admitted for medical treatments in hospital. The current guidelines of the American and European Societies do not recommend routine use of prophylactic anticoagulant treatment in patients receiving chemotherapy, except for myeloma patients on thalidomide, ${ }^{[23-25]}$ partially due to the conflicting results on the efficacy of anticoagulants from clinical trials, the potential risk of increasing bleeding events, and the lack of a suitable anticoagulant that can be easily administered once out of hospital. Therefore, we performed the present meta-analysis based on the latest research to understand the effect and safety of primary DVT prophylaxis in ambulatory patients with solid tumors.

\section{MeTHODS}

\subsection{Inclusion and exclusion criteria}

Studies were included in the present meta-analysis if they met the following criteria: 1) Randomized controlled trials (RCTs) in any language published from Jan 1st, 1993 to Dec 31st, 2015; 2) outpatients with solid tumors; 3 ) control group received either placebo or no treatment at all, was included in this study as comparison with thromboprophylaxis treatment group; 4) outcome measures including incidence of DVT, bleeding events including major bleeding and clinically relevant non-major bleeding, or mortality rate.

Studies were excluded if they met the following criteria: 1) cancer patients without solid tumors, such as myeloma or lymphoma; 2) the incidences of DVT and PE were combined with incidence of venous thromboembolism (VTE) or thrombosis complications; 3) JADAD Score $<3$.

\subsection{Study identification and assessment of study quality} Systematic computerized search of the online databases, including Pubmed, Ovid MEDLINE and EMBASE, was conducted by 2 reviewers independently. The key words used for the search were: "deep vein thrombosis" OR "venous thromboembolism" AND "prophylaxis" OR "prevention” AND "cancer" OR "solid tumor" with each of these short phrases separately. The prophylactic anticoagulants include unfractionated heparin (UFH), low molecular or ultra-low molecular weight heparin (LMWH), direct thrombin inhibitors or direct factor $\mathrm{X}_{a}$ inhibitors. Two reviewers reviewed the titles and abstracts independently, and also searched the bibliographies in the relevant studies for any further potential studies. With the use of JADAD Score, all eligible studies were scored by 2 reviewers independently. To assess the methodological quality of a clinical trial, scale of 0 (very poor) to 7 (rigorous) was used to evaluate studies based on their description of randomization, concealment of allocation, double blinding, and dropouts (withdrawals). ${ }^{[26]}$ Trials with a JADAD score of 1-2 were considered poor, a score of 3 was considered adequate and a score of 4 or higher was considered as high trial quality. Any conflicts between 2 reviewers were resolved after discussion to achieve consensus.

\subsection{Data collection and outcome definition}

The following data for both the thromboprophylaxis treatment group and the control group was extracted from all eligible studies: patient characteristics, number of patients, type of anticoagulants, method of anticoagulants administration, dose of anticoagulants, type of control agents (saline, no thromboprophylaxis, etc.), method of DVT diagnosis, incidence of DVT, incidence of bleeding events including major bleeding and clinically relevant non-major bleeding, as well as mortality rate.

The primary efficacy outcome was primary DVT and survival. The DVT events could be symptomatic or asymptomatic. Diagnosis of DVT could be performed by Doppler imaging, ventilation scan or venography. All recurrent DVT events were excluded. Survival data only include the mortality rate. The end point of primary safety was bleeding, including 
major bleeding and clinically relevant non-major bleeding fusion of blood products, bleeding in critical organs or drop events. Major bleeding events refer to those requiring trans- in haemoglobin $>2 \mathrm{~g}$.

Table 1. Study characteristics

\begin{tabular}{|c|c|c|c|c|c|c|c|c|c|c|}
\hline Study, Year & Types of cancer & $\begin{array}{l}\text { No. of } \\
\text { patients }\end{array}$ & $\begin{array}{l}\text { Tumor type and/or } \\
\text { stage }\end{array}$ & $\begin{array}{l}\text { Types of } \\
\text { anti- } \\
\text { coagulant }\end{array}$ & $\begin{array}{l}\text { Anticoagulant } \\
\text { administration } \\
\text { method }\end{array}$ & $\begin{array}{l}\text { Dose of anti- } \\
\text { coagulant }\end{array}$ & $\begin{array}{l}\text { Control } \\
\text { reagent }\end{array}$ & $\begin{array}{l}\text { DVT } \\
\text { detected } \\
\text { Positions }\end{array}$ & $\begin{array}{l}\text { DVT diagnostic } \\
\text { method }\end{array}$ & $\begin{array}{l}\text { JADAD } \\
\text { score }\end{array}$ \\
\hline $\begin{array}{l}\text { Pelzer } \\
2015^{[27]}\end{array}$ & $\begin{array}{l}\text { Patients with advanced } \\
\text { pancreatic cancer }\end{array}$ & 312 & $\begin{array}{l}\text { Primary 266/312; M0 } \\
\text { 75/312; M1 237/312 }\end{array}$ & LMWH & $\begin{array}{l}\text { Sub- } \\
\text { cutaneously }\end{array}$ & $\begin{array}{l}1 \mathrm{mg} / \mathrm{kg} \text { once } \\
\text { daily for } 3 \\
\text { months }\end{array}$ & No & $\begin{array}{l}\text { Proximal } \\
\text { leg, distal } \\
\text { leg or } \\
\text { upper } \\
\text { extremity }\end{array}$ & $\begin{array}{l}\text { Staging } \\
\text { computed } \\
\text { tomography or } \\
\text { magnetic } \\
\text { resonance } \\
\text { imaging }\end{array}$ & 3 \\
\hline $\begin{array}{l}\text { Macbeth, } \\
2015^{[28]}\end{array}$ & $\begin{array}{l}\text { Patients with primary } \\
\text { bronchial carcinoma }\end{array}$ & 2,202 & $\begin{array}{l}\text { SCLC extensive } \\
\text { 242/392; NSCLC IV } \\
\text { 1009/1810 }\end{array}$ & LMWH & $\begin{array}{l}\text { Sub- } \\
\text { cutaneously }\end{array}$ & $\begin{array}{l}5,000 \text { IU once } \\
\text { daily for a } \\
\text { maximum of } 24 \\
\text { weeks }\end{array}$ & No & N/A & N/A & 3 \\
\hline $\begin{array}{l}\text { Lecumberr, } \\
2013^{[29]}\end{array}$ & $\begin{array}{l}\text { Patients with limited- } \\
\text { stage small cell lung } \\
\text { cancer }\end{array}$ & 38 & Limited-stage & Bemiparin & $\begin{array}{l}\text { Sub- } \\
\text { cutaneously }\end{array}$ & 3,500 $\mathrm{IU}^{*}$ daily & No & N/A ${ }^{* *}$ & N/A & 4 \\
\hline \multirow[b]{2}{*}{$\begin{array}{l}\text { Lavau-Denes, } \\
2013^{[30]}\end{array}$} & \multirow{2}{*}{$\begin{array}{l}\text { Patients with solid } \\
\text { invasive cancer (locally } \\
\text { advanced or metastatic) } \\
\text { with catheter }\end{array}$} & \multirow[b]{2}{*}{407} & \multirow{2}{*}{$\begin{array}{l}\text { Head \& neck 96; Breast } \\
\text { 43; Lung/ Pleura 45; } \\
\text { Colorectal \& Anal 60; } \\
\text { Esophagus \& Stomach } \\
64 \text {; Other digestive 3; } \\
\text { Pancreas \& Biliary tract } \\
\text { 20; Urinary 32; Pelvic } \\
\text { gynecological 19; Other } \\
\text { 14; Primary unknown } 17\end{array}$} & Warfarin & Oral & 1 mg daily & No & \multirow[t]{2}{*}{$\begin{array}{l}\text { Upper } \\
\text { limbs and } \\
\text { cervical } \\
\text { veins }\end{array}$} & \multirow[t]{2}{*}{$\begin{array}{l}\text { Systematic } \\
\text { Doppler } \\
\text { ultrasound }\end{array}$} & \multirow[t]{2}{*}{5} \\
\hline & & & & $\begin{array}{l}\mathrm{LMWH}^{* * *} \\
\text { (dalteparine, } \\
\text { nadroparine, } \\
\text { enoxaparine) }\end{array}$ & $\begin{array}{l}\text { Sub- } \\
\text { cutaneously }\end{array}$ & $\begin{array}{l}\text { Recommended } \\
\text { doses for } \\
\text { prevention }\end{array}$ & & & & \\
\hline $\begin{array}{l}\text { Zwicker, } \\
2013^{[31]}\end{array}$ & $\begin{array}{l}\text { Advanced cancer } \\
\text { patients with high } \\
\text { TFBM, adenocarcinoma } \\
\text { of the pancreas (locally } \\
\text { advanced or metastatic), } \\
\text { colorectal (stage IV), } \\
\text { non-small cell lung } \\
\text { cancer (stage III or IV), } \\
\text { relapsed or stage IV } \\
\text { ovarian, or surgically } \\
\text { unresectable or } \\
\text { metastatic gastric } \\
\text { adenocarcinoma }\end{array}$ & 66 & $\begin{array}{l}\text { Pancreatic 30/66; } \\
\text { non-small cell lung } \\
\text { 21/66; colorctal 15/66 }\end{array}$ & Enoxaparin & $\begin{array}{l}\text { Sub- } \\
\text { cutaneously }\end{array}$ & $\begin{array}{l}40 \mathrm{mg} \text { once } \\
\text { daily }\end{array}$ & No & $\begin{array}{l}\text { Bilateral } \\
\text { lower } \\
\text { extremity }\end{array}$ & Ultrasound & 6 \\
\hline $\begin{array}{l}\text { Haas1, 2012 } \\
\text { (TOPIC-1) }^{[32]}\end{array}$ & $\begin{array}{l}\text { Patients with } \\
\text { disseminated metastatic } \\
\text { breast carcinoma }\end{array}$ & 353 & $\begin{array}{l}\text { Disseminated } \\
\text { metastatic breast } \\
\text { carcinoma } 353\end{array}$ & Certoparin & $\begin{array}{l}\text { Sub- } \\
\text { cutaneously }\end{array}$ & $\begin{array}{l}3,000 \text { IU once } \\
\text { daily, for } 6 \\
\text { months }\end{array}$ & Placebo & N/A & $\begin{array}{l}\text { Venography } \\
\text { and/or } \\
\text { ultrasonography }\end{array}$ & 7 \\
\hline $\begin{array}{l}\text { Haas2, 2012 } \\
\text { (TOPIC-2) }^{[32]}\end{array}$ & $\begin{array}{l}\text { Patients with stage III/IV } \\
\text { non-small-cell lung } \\
\text { carcinoma }\end{array}$ & 546 & Stage IV 289/546 & Certoparin & $\begin{array}{l}\text { Sub- } \\
\text { cutaneously }\end{array}$ & $\begin{array}{l}3,000 \text { IU once } \\
\text { daily, for } 6 \\
\text { months }\end{array}$ & Placebo & N/A & $\begin{array}{l}\text { Venography } \\
\text { and/or } \\
\text { ultrasonography }\end{array}$ & 7 \\
\hline $\begin{array}{l}\text { Agnelli, } \\
2012^{[33]}\end{array}$ & $\begin{array}{l}\text { Patients with metastatic } \\
\text { or locally advanced } \\
\text { cancer of the lung, } \\
\text { pancreas, stomach, colon } \\
\text { or rectum, bladder, or } \\
\text { ovary }\end{array}$ & 3,212 & $\begin{array}{l}\text { Lung 1180; Pancreas } \\
\text { 254; Stomach 411; } \\
\text { Colon/Rectum 925; } \\
\text { Bladder 63; Ovary 279; } \\
\text { Metastatic 2192; Locally } \\
\text { Advanced 1020 }\end{array}$ & Semuloparin & $\begin{array}{l}\text { Sub- } \\
\text { cutaneously }\end{array}$ & $\begin{array}{l}20 \mathrm{mg} \text { once } \\
\text { daily }\end{array}$ & Placebo & $\begin{array}{l}\text { Lower or } \\
\text { upper } \\
\text { limbs }\end{array}$ & N/A & 7 \\
\hline $\begin{array}{l}\text { Levine, } \\
2012^{[34]}\end{array}$ & $\begin{array}{l}\text { Patients with advanced } \\
\text { or metastatic lung, } \\
\text { breast, GI (colon, } \\
\text { rectum, pancreas, } \\
\text { stomach), bladder, } \\
\text { cancer of unknown } \\
\text { origin, ovarian or } \\
\text { prostate cancer, } \\
\text { myeloma or selected } \\
\text { lymphomas }\end{array}$ & 125 & $\begin{array}{l}\text { Breast 32; Lung 12; } \\
\text { Pancreas 15; Stomach 2; } \\
\text { Colon/Rectum 14;Ovary } \\
\text { 2; Prostate 13; Liver } \\
\text { metastases } 29\end{array}$ & Apixaban & Oral & $\begin{array}{l}5 \mathrm{mg} / 10 \mathrm{mg} / \\
20 \mathrm{mg} \text { once } \\
\text { daily for } 12 \\
\text { weeks }\end{array}$ & Placebo & $\begin{array}{l}\text { Proximal } \\
\text { venous } \\
\text { segments, } \\
\text { popliteal } \\
\text { or higher } \\
\text { of the legs }\end{array}$ & $\begin{array}{l}\text { Compression } \\
\text { ultrasound or } \\
\text { venography }\end{array}$ & 7 \\
\hline $\begin{array}{l}\text { Maraveyas, } \\
2012^{[35]}\end{array}$ & $\begin{array}{l}\text { Patients with } \\
\text { non-resectable, recurrent } \\
\text { or metastatic pancreatic } \\
\text { adenocarcinoma }\end{array}$ & 123 & $\begin{array}{l}\text { Locally advanced } \\
\text { 57/123; Metastatic } \\
66 / 123\end{array}$ & Dalteparin & $\begin{array}{l}\text { Sub- } \\
\text { cutaneously }\end{array}$ & $\begin{array}{l}200 \mathrm{IU} / \mathrm{kg} \text { once } \\
\text { daily for } 4 \\
\text { weeks followed } \\
\text { by a step-down } \\
\text { to } 150 \mathrm{IU} / \mathrm{kg} \text { for } \\
\text { a further } 8 \\
\text { weeks }\end{array}$ & No & N/A & N/A & 5 \\
\hline $\begin{array}{l}\text { Tang, } \\
2012{ }^{[36]}\end{array}$ & $\begin{array}{l}\text { Patients with bone tumor } \\
\text { undergoing knee } \\
\text { operation }\end{array}$ & 100 & $\begin{array}{l}\text { Benign 55/100; } \\
\text { Malignant 45/100 }\end{array}$ & Rivaroxaban & Oral & 10 mg daily & No & $\begin{array}{l}\text { Bilateral } \\
\text { lower } \\
\text { extremity }\end{array}$ & Venous Doppler & 3 \\
\hline $\begin{array}{l}\text { Van } \\
\text { Doormaal, } \\
2011^{[37]}\end{array}$ & $\begin{array}{l}\text { Patients with non-small } \\
\text { cell lung cancer (stage } \\
\text { IIIB), hormone- } \\
\text { refractory prostate } \\
\text { cancer or locally } \\
\text { advanced pancreatic } \\
\text { cancer }\end{array}$ & 503 & $\begin{array}{l}\text { Prostate cancer 197/503; } \\
\text { NSCLC 169/503; } \\
\text { Pancreatic cancer } \\
\text { 135/503 }\end{array}$ & Nadroparin & $\begin{array}{l}\text { Sub- } \\
\text { cutaneously }\end{array}$ & $\begin{array}{l}2 \text { weeks } \\
\text { (<50 kg, 3,800 } \\
\text { IU twice daily; } \\
50-70 \mathrm{~kg}, \\
11,400 \mathrm{IU} \text { once } \\
\text { daily; > 70 kg, } \\
15,200 \mathrm{IU} \text { once } \\
\text { daily), followed } \\
\text { by half dose for } \\
\text { an additional } 4 \\
\text { week }\end{array}$ & No & N/A & N/A & 4 \\
\hline $\begin{array}{l}\text { Perry, } \\
2010^{[38]}\end{array}$ & $\begin{array}{l}\text { Patients newly } \\
\text { diagnosed WHO Grade } 3 \\
\text { or Grade } 4 \text { glioma } \\
\text { (anaplastic astrocytoma, } \\
\text { glioblastomamultiforme, } \\
\text { gliosarcoma, anaplastic } \\
\text { oligodendroglioma or } \\
\text { anaplastic mixed glioma) }\end{array}$ & 186 & $\begin{array}{l}\text { Grade } 3 \text { or Grade } 4 \\
\text { glioma }\end{array}$ & Dalteparin & $\begin{array}{l}\text { Sub- } \\
\text { cutaneously }\end{array}$ & $\begin{array}{l}5,000 \text { IU once } \\
\text { daily for } 6 \\
\text { months, up to } \\
12 \text { months }\end{array}$ & Placebo & $\begin{array}{l}\text { Popliteal } \\
\text { or more } \\
\text { proximal } \\
\text { segments } \\
\text { of the } \\
\text { deep veins } \\
\text { of the } \\
\text { lower } \\
\text { limbs }\end{array}$ & $\begin{array}{l}\text { Ascending } \\
\text { venography or } \\
\text { compression } \\
\text { ultrasound }\end{array}$ & 5 \\
\hline $\begin{array}{l}\text { Young AM, } \\
2009^{[39]}\end{array}$ & $\begin{array}{l}\text { Cancer patients with } \\
\text { catheters }\end{array}$ & 812 & $\begin{array}{l}\text { Colorectal 418/812; } \\
\text { Upper gastrointestinal } \\
\text { tract 201/812; Breast } \\
\text { 64/812; Early/no } \\
\text { Residual 264/812 } \\
\text { Advanced 542/812 }\end{array}$ & Warfarin & Oral & $\begin{array}{l}\text { Fixed dose at } \\
1 \text { mg daily or } \\
\text { dose-adjusted } \\
\text { to maintain the } \\
\text { INR }^{* * * *} \\
\text { between } 1.5 \text { and } \\
2.0\end{array}$ & No & N/A & $\begin{array}{l}\text { Venography, } \\
\text { ultrasonography }\end{array}$ & 5 \\
\hline
\end{tabular}

(Table 1 continued on page 38.) 
Table 1. (continued)

\begin{tabular}{|c|c|c|c|c|c|c|c|c|c|c|}
\hline Study, Year & Types of cancer & $\begin{array}{l}\text { No. of } \\
\text { patients }\end{array}$ & $\begin{array}{l}\text { Tumor type and/or } \\
\text { stage }\end{array}$ & $\begin{array}{l}\text { Types of } \\
\text { anti- } \\
\text { coagulant }\end{array}$ & $\begin{array}{l}\text { Anticoagulant } \\
\text { administration } \\
\text { method }\end{array}$ & $\begin{array}{l}\text { Dose of } \\
\text { anti- } \\
\text { coagulant }\end{array}$ & $\begin{array}{l}\text { Control } \\
\text { reagent }\end{array}$ & $\begin{array}{l}\text { DVT } \\
\text { detected } \\
\text { Positions }\end{array}$ & $\begin{array}{l}\text { DVT diagnostic } \\
\text { method }\end{array}$ & $\begin{array}{l}\text { JADAD } \\
\text { score }\end{array}$ \\
\hline $\begin{array}{l}\text { Agnelli, } \\
2009^{[40]}\end{array}$ & $\begin{array}{l}\text { Patients with } \\
\text { metastatic or locally } \\
\text { advanced lung, } \\
\text { gastrointestinal } \\
\text { (stomach, colon or } \\
\text { rectum), pancreatic, } \\
\text { breast, ovarian, or } \\
\text { head and neck } \\
\text { cancer }\end{array}$ & 1,150 & $\begin{array}{l}\text { Lung 279/1150; } \\
\text { Stomach 98/1150; } \\
\text { Colon 235/1150; } \\
\text { Rectum 87/1150; } \\
\text { Pancreas 53/1150; } \\
\text { Breast 165 1150; } \\
\text { Ovary 143/1150; } \\
\text { Head \& neck } \\
\text { 36/1150; Other } \\
\text { 54/1150 }\end{array}$ & Nadroparin & $\begin{array}{l}\text { Sub- } \\
\text { cutaneously }\end{array}$ & $\begin{array}{l}3,800 \text { IU } \\
\text { daily up to } 4 \\
\text { months }\end{array}$ & Placebo & $\begin{array}{l}\text { Lower or } \\
\text { upper limbs }\end{array}$ & N/A & 7 \\
\hline $\begin{array}{l}\text { Shukla, } \\
2008^{[41]}\end{array}$ & $\begin{array}{l}\text { Patients with } \\
\text { colorectal cancer for } \\
\text { curative resection }\end{array}$ & 99 & colorectal cancer & Dalteparin & $\begin{array}{l}\text { Sub- } \\
\text { cutaneously }\end{array}$ & $\begin{array}{l}2,500 \text { IU } \\
\text { once daily }\end{array}$ & No & $\begin{array}{l}\text { Bilateral } \\
\text { external iliac, } \\
\text { common } \\
\text { femoral, } \\
\text { superficial } \\
\text { femoral, } \\
\text { popliteal, and } \\
\text { anterior and } \\
\text { posterior } \\
\text { tibial veins }\end{array}$ & $\begin{array}{l}\text { Duplex } \\
\text { ultrasonography } \\
\text { /Color Doppler }\end{array}$ & 5 \\
\hline $\begin{array}{l}\text { Sideras, } \\
2006^{[42]}\end{array}$ & $\begin{array}{l}\text { Patients with } \\
\text { advanced breast } \\
\text { cancer, prostate } \\
\text { cancer, lung cancer, } \\
\text { colorectal cancer }\end{array}$ & 138 & $\begin{array}{l}\text { Breast 15/138; Colon } \\
\text { 36/138; Postate } \\
\text { 18/138; Small cell } \\
\text { lung 10/138; Non- } \\
\text { small cell lung 59/138 }\end{array}$ & Dalteparin & $\begin{array}{l}\text { Sub- } \\
\text { cutaneously }\end{array}$ & $\begin{array}{l}5,000 \text { IU } \\
\text { once daily }\end{array}$ & $\begin{array}{l}\text { Placebo } \\
\text { /No }\end{array}$ & N/A & N/A & 7 \\
\hline $\begin{array}{l}\text { Klerk, } \\
2005^{[43]}\end{array}$ & $\begin{array}{l}\text { Patients with } \\
\text { advanced } \\
\text { malignancy }\end{array}$ & 302 & $\begin{array}{l}\text { Metastatic disease } \\
\text { 276/302; Locally } \\
\text { advanced 26/302 }\end{array}$ & Nadroparin & $\begin{array}{l}\text { Sub- } \\
\text { cutaneously }\end{array}$ & $\begin{array}{l}3,800 \mathrm{IU}< \\
50 \mathrm{~kg}, 5,700 \\
\mathrm{IU} 50-70 \mathrm{~kg}, \\
7,600 \mathrm{IU}> \\
70 \mathrm{~kg} \text { twice } \\
\text { daily during } \\
\text { the initial } 14 \\
\text { days, and } \\
\text { once daily } \\
\text { thereafter for } \\
\text { another } 4 \\
\text { weeks }\end{array}$ & Placebo & N/A & N/A & 7 \\
\hline $\begin{array}{l}\text { Altinbas, } \\
2004^{[44]}\end{array}$ & $\begin{array}{l}\text { Patients with small } \\
\text { cell lung cancer }\end{array}$ & 84 & $\begin{array}{l}\text { Limited 48/84; } \\
\text { pleural effusion(+) } \\
\text { 12/84; Extensive } \\
\text { disease 36/84; One } \\
\text { metastatic site 28/84; } \\
\text { Two metastatic sites } \\
\text { 8/84 }\end{array}$ & Dalteparin & $\begin{array}{l}\text { Sub- } \\
\text { cutaneously }\end{array}$ & $\begin{array}{l}5,000 \text { IU } \\
\text { once daily } \\
\text { for } 18 \text { weeks }\end{array}$ & No & N/A & N/A & 4 \\
\hline $\begin{array}{l}\text { Kakkar, } \\
2004^{[45]}\end{array}$ & $\begin{array}{l}\text { Patients with } \\
\text { advanced stage III or } \\
\text { IV (locally advanced } \\
\text { or metastatic) } \\
\text { malignant disease of } \\
\text { the breast, lung, } \\
\text { gastrointestinal } \\
\text { tract, pancreas, liver, } \\
\text { genitourinary tract, } \\
\text { ovary, or uterus }\end{array}$ & 374 & $\begin{array}{l}\text { Breast 66/374; } \\
\text { colorectal 70/374; } \\
\text { ovarian 61/374; } \\
\text { pancreatic 42/374; } \\
\text { other 135/374 }\end{array}$ & Dalteparin & $\begin{array}{l}\text { Sub- } \\
\text { cutaneously }\end{array}$ & $\begin{array}{l}5,000 \text { IU } \\
\text { once daily } \\
\text { for } 1 \text { year }\end{array}$ & Placebo & N/A & N/A & 7 \\
\hline $\begin{array}{l}\text { Levine, } \\
1994^{[46]}\end{array}$ & $\begin{array}{l}\text { Patients with } \\
\text { metastatic breast } \\
\text { cancer (stage IV) }\end{array}$ & 311 & $\begin{array}{l}\text { Metastasis: Liver } \\
\text { 101/311; Lung } \\
\text { 97/311; Brain 1/311; } \\
\text { Bone only 58/311; } \\
\text { Regional nodes only } \\
\text { 24/311; Chest wall } \\
\text { only 9/311 }\end{array}$ & Warfarin & Oral & $\begin{array}{l}1 \mathrm{mg} / \mathrm{kg} \text { once } \\
\text { daily for } 6 \\
\text { weeks }\end{array}$ & Placebo & N/A & $\begin{array}{l}\text { Duplex } \\
\text { ultrasonography } \\
\text { or venography }\end{array}$ & 5 \\
\hline $\begin{array}{l}\text { Marassii, } \\
1993^{[47]}\end{array}$ & $\begin{array}{l}\text { Cancer patients with } \\
\text { major abdominal } \\
\text { oncological surgery }\end{array}$ & 61 & $\begin{array}{l}\text { Gastric 19; Ileal 3; } \\
\text { Colonic 35; other } 4\end{array}$ & $\begin{array}{l}\text { LMWH } \\
\text { (Seleparina, } \\
\text { CY 216) }\end{array}$ & $\begin{array}{l}\text { Sub- } \\
\text { cutaneously }\end{array}$ & $\begin{array}{l}3,825 \text { IU } \\
\text { twice daily }\end{array}$ & No & N/A & $\begin{array}{l}125 \text { I-labelled } \\
\text { fibrinogen leg } \\
\text { scan }\end{array}$ & 4 \\
\hline
\end{tabular}

Note. ${ }^{*}$ IU: International unit; ${ }^{* *} \mathrm{~N} / \mathrm{A}$ : Information not available from the reference; ${ }^{* * *}$ LMWH: Low molecular weight heparin; ${ }^{* * * *}$ INR: International normalized ratio.

\subsection{Statistical analysis}

Continuous data for each arm in a particular study was expressed as the mean and standard deviation (SD), and the treatment effect as the mean differences. Dichotomous data for each arm in a particular study was expressed as proportions or risks, and the treatment effect as risk differences. Relevant data was analyzed using RevMan 5.0. Heterogeneity was explored by Chi-squared test with a significance set at a $p$ value of .05 , and the quantity of heterogeneity was measured by $\mathrm{I}^{2}$. The statistic $\mathrm{I}^{2}$ value is a measure of the percentage of variation in the data that is as a result of heterogeneity as opposed to chance. $\mathrm{I}^{2}$ values of $0-25 \%$ are considered low, $25 \%-75 \%$ as moderate, while values over $75 \%$ are considered high heterogeneity. ${ }^{[22]}$ For moderate heterogeneity, the fixed effects model was used; the random effects model was used when the heterogeneity was high.

\section{RESULTS}

\subsection{Literature search and study characteristics}

A total of 1,410 potentially relevant articles were published from the databases, among which 1,376 were excluded according to our inclusion and exclusion criteria from the titles, abstracts and/or articles. Among the remaining 35 trials in the 34 articles, 13 were excluded due to the JADAD score $<$ 3. Therefore, a total of 22 trials in 21 eligible studies ${ }^{[27-47]}$ were included in the final analysis, one of which was written in Chinese ${ }^{[36]}$ and all of the others were written in English. The key information of the study and patient characteristics was included in the present meta-analysis (see Tables 1 and 2). 
Table 2. Patients' characteristics

\begin{tabular}{|c|c|c|c|c|c|c|c|c|}
\hline \multirow[t]{2}{*}{ Author (Year) } & \multicolumn{2}{|c|}{ Patient number } & \multicolumn{2}{|c|}{ Gender (Male) } & \multicolumn{2}{|c|}{ Mean age (years, SD or range) } & \multicolumn{2}{|c|}{$\begin{array}{l}\text { Mean BMI }\left(\mathrm{kg} / \mathrm{m}^{2}\right) \text { or weight }(\mathrm{kg}) \\
\text { (SD or range) }\end{array}$} \\
\hline & AC & Con & AC & Con & AC & Con & AC & Con \\
\hline Pelzer, $2015^{[27]}$ & 160 & 152 & 91 & 94 & $62.0(32.0-81.0)$ & $63.0(27.0-83.0)$ & $24.3(15.2-43.0)$ & $23.8(16-39.2)$ \\
\hline $\begin{array}{l}\text { Macbeth, } \\
2015^{[28]}\end{array}$ & 1,101 & 1,101 & 661 & 656 & $65.0(59.0-71.0)$ & $64.0(58.0-71.0)$ & $25.6(22.9-29.0)$ & $25.8(22.7-29.1)$ \\
\hline $\begin{array}{l}\text { Lecumberri, } \\
2013^{[29]}\end{array}$ & 20 & 18 & 17 & 16 & $61.1 \pm 7.5$ & $64.5 \pm 10.0$ & $74.1 \pm 12.1$ & $77.1 \pm 20.1$ \\
\hline $\begin{array}{l}\text { Lavau-Denes, } \\
2013^{[30] \text { a }}\end{array}$ & $\begin{array}{l}134 \\
138\end{array}$ & 135 & $\begin{array}{l}81 \\
78\end{array}$ & 84 & $\begin{array}{l}59.0 \pm 10.9 \\
61.0 \pm 10.6\end{array}$ & $60.0 \pm 11.8$ & $\begin{array}{l}\text { N/A*** } \\
\text { N/A }\end{array}$ & N/A \\
\hline $\begin{array}{l}\text { Zwicker, } \\
2013^{[31] \mathrm{b}}\end{array}$ & 23 & $\begin{array}{l}11 \\
32\end{array}$ & 14 & $\begin{array}{l}5 \\
19\end{array}$ & $68.1(46.6-80.1)$ & $\begin{array}{l}67.5(28.8-78.7) \\
62.8(42.7-83.8)\end{array}$ & $23.8(16.6-31.6)$ & $\begin{array}{l}23.8(20.0-34.4) \\
26.3(19.0-48.7)\end{array}$ \\
\hline $\begin{array}{l}\text { Haas1, 2012 } \\
\text { (TOPIC-1) }^{[32]}\end{array}$ & 174 & 177 & N/A & N/A & $54.6 \pm 10.3$ & $56.6 \pm 11.0$ & $27.0 \pm 4.9$ & $27.5 \pm 5.7$ \\
\hline $\begin{array}{l}\text { Haas2, } 2012 \\
\text { (TOPIC-2) }^{[32]}\end{array}$ & 268 & 264 & 227 & 227 & $60.8 \pm 9.5$ & $60.3 \pm 10.0$ & $24.7 \pm 4.1$ & $24.6 \pm 4.2$ \\
\hline $\begin{array}{l}\text { Agnelli, } \\
2012^{[33]}\end{array}$ & 1,608 & 1,604 & 974 & 956 & $59.8 \pm 10.6$ & $59.4 \pm 10.6$ & $24.9 \pm 5.1$ & $24.7 \pm 4.9$ \\
\hline $\begin{array}{l}\text { Levine, } \\
2012^{[34] \text { c }}\end{array}$ & $\begin{array}{l}32 \\
30 \\
33\end{array}$ & 30 & $\begin{array}{l}15 \\
13 \\
20\end{array}$ & 15 & $\begin{array}{l}57.0(41.0-67.0) \\
60.0(39.0-76.0) \\
64.0(25.0-86.0)\end{array}$ & $59.0(20.0-82.0)$ & N/A & N/A \\
\hline $\begin{array}{l}\text { Maraveyas, } \\
2012^{[35]}\end{array}$ & 60 & 63 & 36 & 36 & $62.0(40.0-79.0)$ & $66.0(43.0-82.0)$ & N/A & N/A \\
\hline Tang, $2012^{[36]}$ & 50 & 50 & 26 & 28 & $33.7 \pm 14.4$ & $35.1 \pm 16.6$ & N/A & N/A \\
\hline $\begin{array}{l}\text { vanDoormaal, } \\
2011^{[37]}\end{array}$ & 244 & 259 & 197 & 206 & $65.0 \pm 10.0$ & $65.0 \pm 9.8$ & $74.3 \pm 15.5$ & $73.2 \pm 14.2$ \\
\hline Perry, $2010^{[38]}$ & 99 & 87 & 61 & 50 & $57.0(30.0-81.0)$ & $55.0(26.0-77.0)$ & N/A & N/A \\
\hline $\begin{array}{l}\text { Young AM, } \\
2009^{[39]}\end{array}$ & 408 & 404 & 252 & 247 & $60.0(53.0-68.0)$ & $61.0(53.0-68.0)$ & N/A & N/A \\
\hline $\begin{array}{l}\text { Agnelli, } \\
2009^{[40]}\end{array}$ & 769 & 381 & 372 & 183 & $62.1 \pm 10.3$ & $63.7 \pm 9.2$ & $25.4 \pm 4.4$ & $25.2 \pm 4.2$ \\
\hline $\begin{array}{l}\text { Shukla, } \\
2008^{[41]}\end{array}$ & 51 & 48 & N/A & N/A & N/A & N/A & N/A & N/A \\
\hline Sideras, & 24 & 26 & 12 & 11 & 64.5 & 63.5 & N/A & N/A \\
\hline $\begin{array}{l}2006^{[42] \mathrm{d}} \\
\text { Klerk, } 2005^{[43]}\end{array}$ & $\begin{array}{l}44 \\
148\end{array}$ & $\begin{array}{l}44 \\
154\end{array}$ & $\begin{array}{l}28 \\
77\end{array}$ & $\begin{array}{l}31 \\
81\end{array}$ & $\begin{array}{l}68.5 \\
63.0(36.0-86.0)\end{array}$ & $\begin{array}{l}70.5 \\
64.0(28.0-83.0)\end{array}$ & $71.0(40.0-135)$ & $69.0(43.0-96.0)$ \\
\hline $\begin{array}{l}\text { Altinbas, } \\
2004^{[44]}\end{array}$ & 42 & 42 & 33 & 35 & $57.5(34.0-74.0)$ & $58.0(37.0-75.0)$ & N/A & N/A \\
\hline $\begin{array}{l}\text { Kakkar, } \\
2004^{[45]}\end{array}$ & 190 & 184 & 77 & 84 & $62.0(53.8-68.4)$ & $60.9(52.4-69.4)$ & N/A & N/A \\
\hline $\begin{array}{l}\text { Levine, } \\
1994^{[46]}\end{array}$ & 152 & 159 & N/A & N/A & $57.1 \pm 10.2$ & $56.1 \pm 10.9$ & N/A & N/A \\
\hline $\begin{array}{l}\text { Marassii, } \\
1993^{[47]}\end{array}$ & 31 & 31 & N/A & N/A & N/A & N/A & N/A & N/A \\
\hline
\end{tabular}

Note. ${ }^{*} \mathrm{AC}$, anticoagulant group, ${ }^{* *}$ Con, control group, ${ }^{* * *} \mathrm{~N} / \mathrm{A}$, information not available from the reference. ${ }^{\text {a }}$ upper line: warfarin group; lower line: LMWH group. ${ }^{\mathrm{b}}$ upper line: high plasma concentration of tissue factor-bearing microparticles (TFBM); lower line: low plasma concentrion of TFBM. ${ }^{\mathrm{C}}$ upper line: 5 mg apixaban; middle line: $10 \mathrm{mg}$ apixaban; lower line: $20 \mathrm{mg}$ apixaban. ${ }^{\mathrm{d}}$ upper line: blinded LMWH vs. placebo; lower line: unblended LMWH vs. standard care.

The number of patients in these trials ranged from 34 to 3,212. All of them were with solid tumors. A total of 6,033 patients in the prophylactic group and 5,456 patients in the control group were included. The median age of participants in these trials was between 33.7 to 70.5 years old, and was similar in these 2 groups. In 3 articles warfarin was used for primary prophylactic anticoagulant, in another 2 trials, apixaban or rivaroxaban was used as primary thromboprophylaxis, and the rest low molecular weight heparin (LMWH) was sub- cutaneously injected as primary interventions, with placebo as control reagent if there is one.

\subsection{Incidence of DVT in patients treated with prophylac- tic anticoagulants}

Information on the incidence of DVT in patients with solid tumors was reported in 16 trials, including 5,304 patients in the prophylactic group and 4,703 patients in the control group. A total of 270 DVT events were identified in the pro- 
phylactic group (89) and the control group (181). DVT was identified in bilateral lower or upper limbs, cervical veins, or more proximal venous segments. The incidence of DVT in cancer patients treated with prophylactic anticoagulants was 0 to $12 \%$, and that in the control group was 0 to $35 \%$. The pooled DVT incidence rates were $1.68 \%(89 / 5,304)$ and $3.85 \%(181 / 4,703)$ for the prophylactic group and control group, respectively (see Figure 1a). Only one study in which prophylactic treatment produced worse outcome reported a relevant publication bias (van Doormaal 2011, [37] see Figure 1b). Treatment with prophylactic anticoagulants to patients with solid tumors significantly reduced the DVT incidence rate $(p<.00001, \mathrm{RR}=0.45,95 \% \mathrm{CI}: 0.36-0.58)$, without statistically significant heterogeneity between trials $(p=.11$, $\left.\mathrm{I}^{2}=33 \%\right)$.

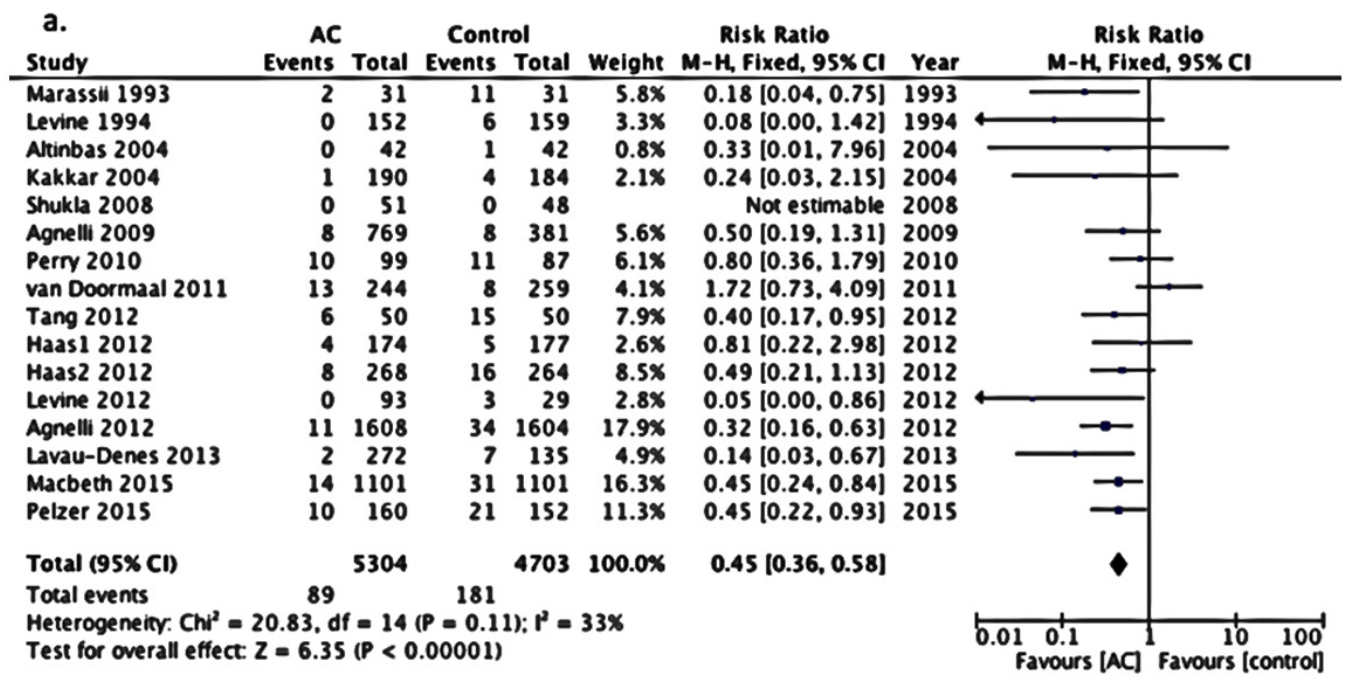

b.

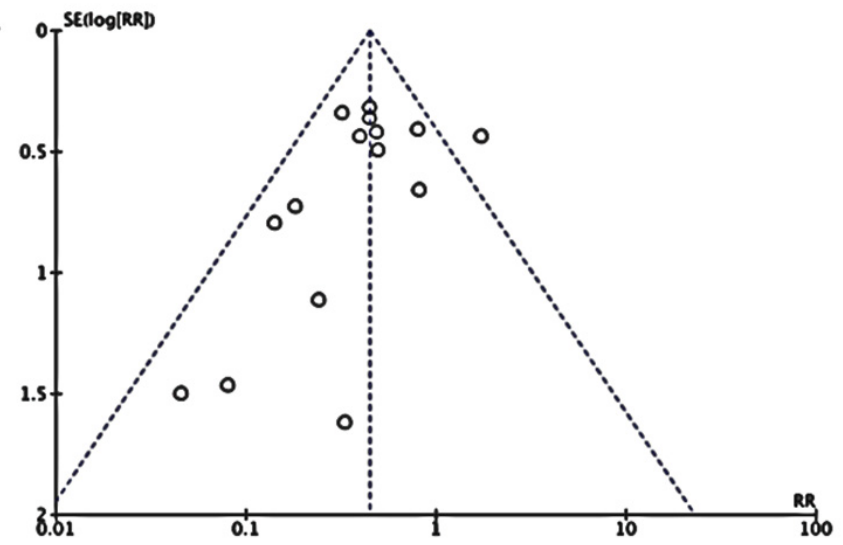

Figure 1. a) The prophylactic anticoagulants significantly reduced the DVT incidence in patients with solid tumors. b) Funnel plot corresponding to primary analysis of DVT prevention in patients with solid tumors.

\subsection{Incidence of bleeding events treated with prophylac- tic anticoagulants}

Eighteen trials with data on major bleeding were analyzed for statistical significance. A total of 5,629 patients were in the prophylactic group and 5,187 in the control group. A total of 170 major bleeding events were reported, with 99 events in the prophylactic group and 71 events in the control group, respectively. The incidence of major bleedings in patients treated with prophylactic anticoagulants was between 0 and $8.13 \%$, and that in the control group was between 0 and
$7.14 \%$. The pooled incidence rates of major bleedings were $1.76 \%(99 / 5,629)$ and $1.37 \%(71 / 5,187)$ for the prophylactic and control group, respectively (see Figure 2a). We did not detect a relevant publication bias in the present analysis (see Figure 2b). Treatment with prophylactic anticoagulants to patients with solid tumors did not significantly increase the major bleeding rate ( $p=.06, \mathrm{RR}=1.33,95 \% \mathrm{CI}$ : 0.99-1.79), without statistically significant heterogeneity between trials $\left(p=.71, \mathrm{I}^{2}=0 \%\right)$. 


\begin{tabular}{|c|c|c|c|c|c|c|c|c|c|}
\hline $\begin{array}{l}\text { a. } \\
\text { Study }\end{array}$ & \multicolumn{2}{|l|}{$\mathrm{AC}$} & \multicolumn{2}{|c|}{ Control } & \multicolumn{3}{|c|}{ Risk Ratio } & \multicolumn{2}{|c|}{$\begin{array}{c}\text { Risk Ratio } \\
\text { M-H, Fixed, 95\% Cl }\end{array}$} \\
\hline Levine 1994 & 1 & 152 & 2 & 159 & $2.6 \%$ & $0.52[0.05,5.71]$ & 1994 & & \\
\hline Kakkar 2004 & 1 & 190 & 0 & 184 & $0.7 \%$ & $2.91[0.12,70.87]$ & 2004 & & \\
\hline Klerk 2005 & 5 & 148 & 1 & 154 & $1.3 \%$ & $5.20[0.62,44.01]$ & 2005 & & \\
\hline Sideras 2006 & 2 & 68 & 5 & 70 & $6.6 \%$ & $0.41[0.08,2.05]$ & 2006 & & \\
\hline Agnelli 2009 & 5 & 769 & 0 & 381 & $0.9 \%$ & $5.46[0.30,98.43]$ & 2009 & & \\
\hline Young AM 2009 & 7 & 408 & 1 & 404 & $1.4 \%$ & $6.93[0.86,56.08]$ & 2009 & & \\
\hline Perry 2010 & 5 & 99 & 1 & 87 & $1.4 \%$ & $4.39[0.52,36.89]$ & 2010 & & \\
\hline van Doormaal 2011 & 10 & 244 & 9 & 259 & $11.8 \%$ & $1.18[0.49,2.85]$ & 2011 & & \\
\hline Agnelli 2012 & 19 & 1598 & 18 & 1583 & $24.3 \%$ & $1.05[0.55,1.98]$ & 2012 & & \\
\hline Haas1 2012 & 3 & 174 & 0 & 178 & $0.7 \%$ & $7.16[0.37,137.60]$ & 2012 & & \\
\hline Tang 2012 & 1 & 50 & 0 & 50 & $0.7 \%$ & $3.00[0.13,71.92]$ & 2012 & & \\
\hline Maraveyas 2012 & 2 & 59 & 2 & 62 & $2.6 \%$ & $1.05[0.15,7.22]$ & 2012 & & \\
\hline Levine 2012 & 2 & 93 & 1 & 29 & $2.1 \%$ & $0.62[0.06,6.63]$ & 2012 & & \\
\hline Haas2 2012 & 10 & 273 & 6 & 273 & $8.1 \%$ & $1.67[0.61,4.52]$ & 2012 & & \\
\hline Zwicker 2013 & 0 & 23 & 1 & 43 & $1.4 \%$ & $0.61[0.03,14.43]$ & 2013 & & \\
\hline Lecumberri 2013 & 0 & 20 & 1 & 18 & $2.1 \%$ & $0.30[0.01,6.97]$ & 2013 & & \\
\hline Pelzer 2015 & 13 & 160 & 10 & 152 & $13.8 \%$ & $1.24[0.56,2.73]$ & 2015 & & \\
\hline Macbeth 2015 & 13 & 1101 & 13 & 1101 & $17.5 \%$ & $1.00[0.47,2.15]$ & 2015 & & \\
\hline Total $(95 \% \mathrm{CI})$ & & 5629 & & 5187 & $100.0 \%$ & $1.33[0.99,1.79]$ & & & \\
\hline Total events & 99 & & 71 & & & & & & \\
\hline $\begin{array}{l}\text { Heterogeneity: } \mathrm{Chi}^{2}= \\
\text { Test for overall effec }\end{array}$ & $\begin{array}{l}13.38, d \\
Z=1.89\end{array}$ & $\begin{array}{l}f=17 \\
(P=0\end{array}$ & $\begin{array}{l}(P=0.7 \\
.06)\end{array}$ & 1); $1^{2}=$ & & & & \begin{tabular}{|lc|}
0.01 & \multicolumn{1}{|c|}{0.1} \\
Favours [AC]
\end{tabular} & $\begin{array}{r}10 \\
\text { Favours [c }\end{array}$ \\
\hline
\end{tabular}

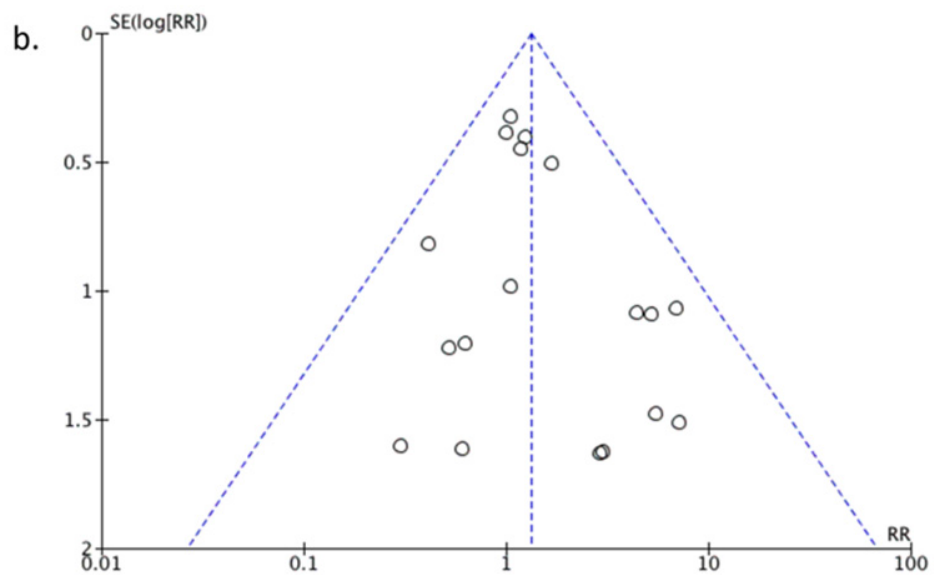

Figure 2. a) The prophylactic anticoagulants didn't cause significant risk of major bleedings in patients with solid tumors. b) Funnel plot corresponding to primary analysis of major bleeding occurrence in patients with solid tumors.

Fifteen trials were enrolled for statistical analysis of clinically relevant non-major bleeding events. A total of 5,321 patients were in prophylactic group and 4,877 in control group. In these studies, 316 non-major bleedings including 219 in the prophylactic group and 97 in the control group were reported. The incidence of non-major bleedings in patients treated with prophylactic anticoagulants was between $0.74 \%$ and $10.00 \%$, and that in the control group was between 0 and $22.22 \%$. The pooled incidence rates were $4.12 \%(219 / 5,321)$ and $1.99 \%$ $(97 / 4,877)$ for the prophylactic and control patients, respectively (see Figure 3a). Statistical significance was observed between the 2 groups regarding the incidence of clinically relevant non-major bleeding events $(p<.00001, \mathrm{RR}=1.83$, 95\%CI: 1.46-2.30), with moderate heterogeneity between trials $\left(p=.01, \mathrm{I}^{2}=52 \%\right)$.

\subsection{Mortality rate of patients treated with prophylactic anticoagulants}

To assess the mortality rate, 5,297 prophylactic patients and 4,718 control patients from 16 trials were analyzed. 4,396 patients including 2,253 prophylactic patients and 2,143 control patients died during the follow-up period. The mortality rate in cancer patients treated with prophylactic anticoagulants was between 0 and $60.81 \%$, and that in control patients was from 0 to $72.73 \%$. The pooled mortality rate was $42.53 \%$ $(2,253 / 5,297)$ and $45.42 \%(2,143 / 4,718)$ for the prophylactic and control groups, respectively (see Figure 4a). Although after prophylactic anticoagulant treatment, the mortality rate of the patients with solid tumors was not statistically significant ( $p=.22, \mathrm{RR}=0.97,95 \% \mathrm{CI}: 0.93-1.02)$, the overall mortality rate of the prophylactic patients was still lower than that of the control. Moderate heterogeneity between trials was found $\left(p=.05, \mathrm{I}^{2}=40 \%\right)$. 


\begin{tabular}{|c|c|c|c|c|c|c|c|c|c|}
\hline $\begin{array}{c}\text { a. } \\
\text { Study }\end{array}$ & \multicolumn{2}{|l|}{$\mathrm{AC}$} & \multicolumn{2}{|c|}{ Control } & \multicolumn{3}{|c|}{ Risk Ratio } & \multicolumn{2}{|c|}{$\begin{array}{c}\text { Risk Ratio } \\
\text { M-H, Fixed, 95\% Cl }\end{array}$} \\
\hline Levine 1994 & 7 & 152 & 3 & 159 & $2.7 \%$ & $2.44[0.64,9.27]$ & 1994 & & \\
\hline Altinbas 2004 & 1 & 42 & 0 & 42 & $0.5 \%$ & $3.00[0.13,71.61]$ & 2004 & & \\
\hline Klerk 2005 & 5 & 148 & 0 & 154 & $0.4 \%$ & $11.44[0.64,205.14]$ & 2005 & & \\
\hline Young AM 2009 & 3 & 408 & 0 & 404 & $0.5 \%$ & $6.93[0.36,133.76]$ & 2009 & & \\
\hline Agnelli 2009 & 57 & 769 & 30 & 381 & $36.7 \%$ & $0.94[0.62,1.44]$ & 2009 & & \\
\hline Maraveyas 2012 & 5 & 59 & 2 & 62 & $1.8 \%$ & $2.63[0.53,13.02]$ & 2012 & & \\
\hline Levine 2012 & 4 & 93 & 0 & 29 & $0.7 \%$ & $2.87[0.16,51.82]$ & 2012 & & \\
\hline Tang 2012 & 2 & 50 & 2 & 50 & $1.8 \%$ & $1.00[0.15,6.82]$ & 2012 & & \\
\hline Haas1 2012 & 6 & 174 & 3 & 178 & $2.7 \%$ & $2.05[0.52,8.05]$ & 2012 & & \\
\hline Haas2 2012 & 27 & 273 & 14 & 273 & $12.8 \%$ & $1.93[1.03,3.60]$ & 2012 & & 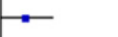 \\
\hline Agnelli 2012 & 26 & 1598 & 14 & 1583 & $12.9 \%$ & $1.84[0.96,3.51]$ & 2012 & & $\because$ \\
\hline Total events & 219 & & 97 & & & & & & \\
\hline $\begin{array}{l}\text { Heterogeneity: } \mathrm{Chi}^{2}= \\
\text { Test for overall effect }\end{array}$ & $\begin{array}{l}28.95, d \\
Z=5.20\end{array}$ & $\begin{array}{l}f=14 \\
(P<0\end{array}$ & $\begin{array}{l}(P=0.01 \\
.00001)\end{array}$ & 1); $\left.\right|^{2}=$ & $52 \%$ & & & $\begin{array}{ll}0.01 & 0.1 \\
& \text { Favours }\end{array}$ & $\begin{array}{r}10 \\
\text { Favours [c }\end{array}$ \\
\hline
\end{tabular}

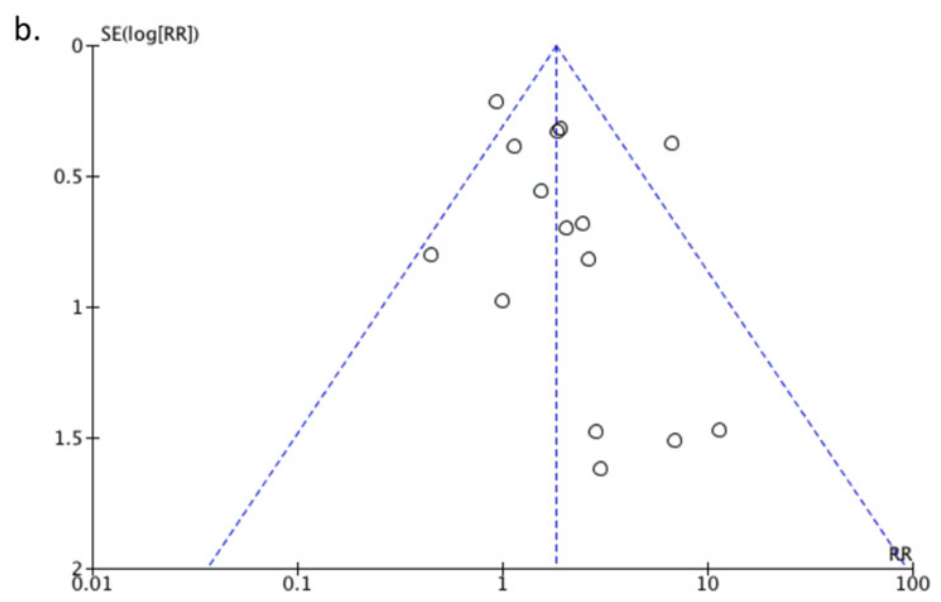

Figure 3. a) Statistical difference between the prophylactic group and the control group was observed regarding the incidence of minor bleedings in patients with solid tumors. b) Funnel plot corresponding to primary analysis of clinical relevant non-major bleeding occurrence in patients with solid tumors.

\section{Discussion}

Cancer treatment, especially chemotherapy, is always associated with an activation of haemostatic system. ${ }^{[48]}$ The underlying mechanism of prothrombosis caused by cancer itself and various treatments is not clear. Over the last few decades, only a few studies have been focused on it. ${ }^{[15]}$

Due to the fact that cancer patients have high risk to develop venous thrombosis because of the activation of haemostatic system during cancer progression, ${ }^{[2]}$ many cohort or prospective studies investigated the side effects of prophylactic anticoagulants, most of which were heparin-based interventions. ${ }^{[27-47]}$ The inhibitory effect on cancer progression and the survival by low molecular weight heparin was first reported in 1992 by Prandoni et al. They found a significant reduction of overall mortality rate among cancer patients with VTE who took LMWH treatment. ${ }^{[49]}$ However, partially due to the conflicting results from clinical trials and the fear of bleeding, the current guidelines of the American and European Societies do not recommend routine use of prophylactic anticoagulant treatment in ambulatory patients who receive chemotherapy, except for myeloma patients on thalidomide. ${ }^{[23-25]}$ Therefore it is necessary to perform the present meta-analysis based on the latest research to investigate the efficacy and safety of primary DVT prophylaxis in patients with solid tumors.

In this study, we analyzed 22 randomized controlled trials. The effects of antithrombotic prophylaxis on the incidence rates of DVT and bleedings, as well as the mortality rate in cancer patients were analyzed. We found that the pooled DVT incidence rates were $1.68 \%$ ( 89 events in 5,304 patients) for the prophylactic patients and $3.85 \%$ (181 events in 4,703 patients) for the control patients. Compared with placebo or no anticoagulants, a $56.36 \%$ reduction in DVT events with the use of prophylactic anticoagulants was observed. Overall, 
our analysis showed that thromboprophylaxis significantly reduced the episodes of DVT in patients with solid tumors $(p<.00001)$. Regarding bleeding, the pooled incidence rates of major bleeding events and clinically relevant non-major bleeding events were $1.76 \%$ (99 events in 5,629 patients) and $4.12 \%$ (219 events in 5,321 patients) in the prophylactic group, respectively; and were $1.37 \%$ (71 events in 5,187 patients) and $1.99 \%$ (97 events in 4,877 patients) in the control group, respectively. Our analysis showed that thromboprophylaxis did not have significant impact on the incidence rate of major bleedings $(p=.06)$. Although the difference on the incidence rates of clinically relevant non-major bleedings between the 2 groups was statistically significant ( $p<.00001)$, all these bleedings did not locate in critical organs and were all well-controlled. Additionally, although the mortality benefit derived from thromboprophylaxis was not statistically significant ( $p=.22$ ), the overall mortality rate in the prophylactic group was still lower than that in the control group ( $42.53 \%$ and $45.42 \%$, respectively). The heterogeneity of the mortality rate among studies was high, which might partially be due to the different prognosis of various solid tumors. Therefore, to minimize the heterogeneity of the studies, the mortality rate was evaluated with the data at 1-year follow-up period. If the study did not specify the follow-up time point or the follow up is $<1$ year, the mortality rate was evaluated with the data during the entire follow-up period.

The total events in this meta-analysis were more than previously published events, and there were 3 key aspects in our analysis: 1) we only included solid tumor malignancies, 2) we only included ambulatory cancer patients, 3) and we only focused on incidence rates of DVT instead of all kinds of venous thromboembolism.

As for mortality rate of patients with solid tumors, we did not observe a significant difference between prophylactic and control patients $(p=.22)$. The lack of significant benefit on survival rate in current analysis might be attributed to the controversial results from the involved studies. Several clinical trials claimed that low-molecular weight heparin could prolong the survival of cancer patients. ${ }^{[45-47]}$ While two studies from Agnelli G et al., ${ }^{[33,40]}$ which were with 2 of the 3 highest weights in the analysis (9.4\% and $32.6 \%$, respectively), showed no beneficial effects of prophylactic anticoagulants on survival. Several factors may cause such results in their studies. First, the duration of prophylactic anticoagulant treatment in their study was shorter. Second, the dose of low-molecular-weight heparin, and the prophylactic anticoagulant in their study was lower than the doses showing a favorable effect on survival. Third, the patients included in their studies were with metastatic or locally advanced disease, whereas most of the benefit from low-molecular-weight hep- arin noted in survival studies has been observed in patients with less advanced disease. Nonetheless, the overall mortality rate of the prophylactic group was still lower than that of the control group ( $42.53 \%$ vs. $45.42 \%)$, which suggested a beneficial trend of prophylactic anticoagulant treatment. Further studies with longer duration and higher dose of treatment will be required to confirm the effects of prophylactic anticoagulant treatment on the survival of cancer patients.

To date, various kinds of anticoagulants have been developed, including heparin, different types of low molecular weight heparin, warfarin, vitamin $\mathrm{K}$ antagonists, and new oral anticoagulants. Among these anticoagulants, heparin or low molecular weight heparin is still used as most primary intervention. Among the 22 trials included in this meta-analysis, 18 studies used low molecular weight heparin as primary prophylactic anticoagulant intervention. Several mechanisms were ever proposed for the antitumor effects of heparins, such as induction of apoptosis, inhibition of tumor cell proliferation, angiogenesis and extracellular matrix remodeling, prevention of metastatic spreading by interfering the adhesion of cancer cells to the endothelium. ${ }^{[20,51,52]}$ For instance, the ABEL study (Adjuvant Bemiparin in Small Cell Lung Cancer) ${ }^{[29]}$ supported the hypothesis that the main anti-tumor effects of bemiparin in vivo might be the prevention of distant metastasis of the tumor cells rather than direct cytoreduction, since the benefit in terms of survival observed in patients receiving low molecular weight heparin was not associated with a better response rate to chemoradiotherapy. ${ }^{[29]}$

However, most thromboprophylactic drugs available currently are not ideal for DVT prophylaxis, because they can not be easily administered once patients are out of hospital. And this may also contribute to the fact that prophylactic anticoagulants are not routinely used to cancer patients with chemotherapy treatment. For instance, unfractionated heparin and low molecular weight heparin require subcutaneous injection every day. Warfarin is also difficult to be administered since it will induce nausea, vomiting, poor nutrition and interaction with other medications. Therefore, new oral anticoagulants, such as apixaban, which is a new factor $\mathrm{X}_{a}$ inhibitor targeting the active site of factor $\mathrm{X}_{a}$ without requiring antithrombin III, might be a better choice for extended DVT prophylaxis in cancer patients with chemotherapy. These new oral anticoagulants are administered orally and do not require laboratory monitoring and dose adjustment. Additionally, new oral anticoagulants have shorter half-life, which could facilitate temporary interruptions for invasive procedures or when thrombocytopenia occurs. ${ }^{[53]}$ One recent meta-analysis compared the efficacy and safety of new oral anticoagulants with vitamin $\mathrm{K}$ antagonists in patients with cancer-associated acute venous thromboembolism. ${ }^{[54]}$ It showed that both ef- 
ficacy and safety of new oral anticoagulants in treatment of cancer-associated acute symptomatic venous thromboembolism was at least comparable to those of vitamin $\mathrm{K}$ antagonists. Although new oral anticoagulants are yet not be recommended as the first-line treatment for venous throm- boembolism in cancer patients compared with low molecular weight heparin, these new oral anticoagulants have more apparent advantages. Therefore, more prospective clinical trials for the evaluation of their efficacy and safety among cancer patients are urgently required in the near future.

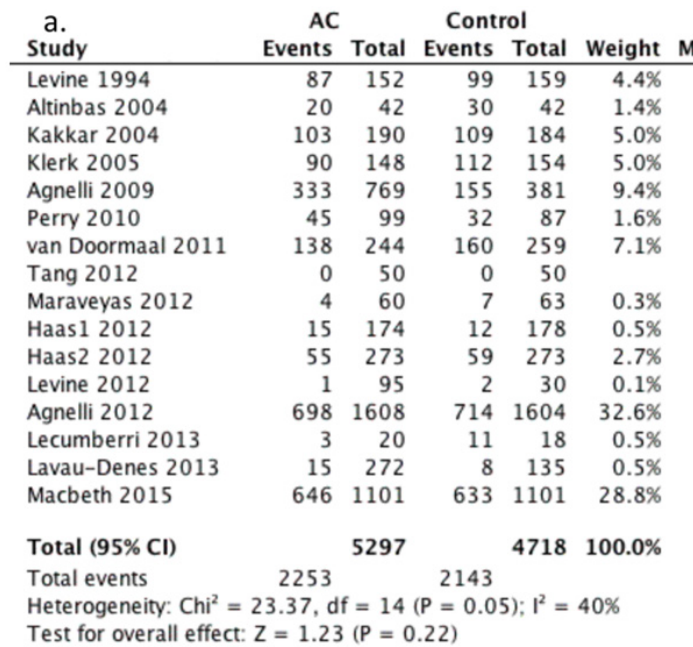

Risk Ratio Risk Ratio $\mathrm{M}-\mathrm{H}$, Fixed, 95\% Cl Year $\mathrm{M}-\mathrm{H}$, Fixed, $95 \% \mathrm{Cl}$

b.

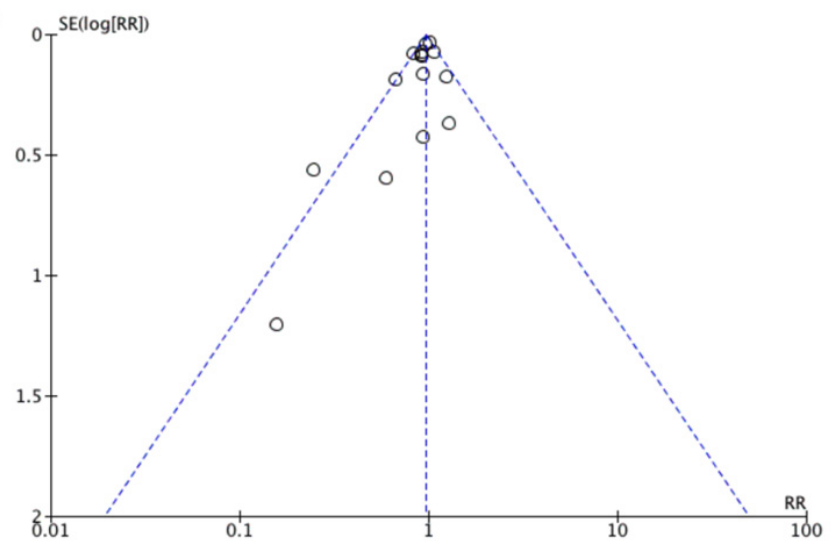

Figure 4. a) Forest plot diagram showing the effect of prophylactic anticoagulants on the mortality rate of patients with solid tumors. b Funnel plot corresponding to primary analysis of mortality in patients with solid tumors.

\section{Conclusions}

The present meta-analysis demonstrated that prophylactic anticoagulants in cancer patients could significantly reduce the incidence rates of DVT, while had no significantly impact on the incidence rate of major bleeding events but increased the incidence of clinically-relevant non-major bleedings. Our results suggest that the prophylactic anticoagulants could be used for cancer patients without major bleeding risks.

\section{ACKNOWLEDGements}

This work was supported by Talent Cultivation Plan of Fifth People's Hospital of Shanghai, P.R.China (Grant No.2013YYJRC14) and by Health and Family Planning Commission of Minhang District, Shanghai, P.R.China (Grant No.2014MW06).

\section{CONFLICTS OF INTEREST DISClOSURE}

The authors declare that there is no conflict of interest statement.

\section{REFERENCES}

[1] Cronan JJ, Dorfman GS, Grusmark J. Lower-extremity deep venous thrombosis: further experience with and refinements of US assessment. Radiology. 1988; 168: 101-7. PMid:2967984. https : //doi.org/10.1148/radiology.168.1.2967984

[2] Prandoni P. Antithrombotic strategies in patients with cancer. Throm- 
bosis and Haemostasis. 1997; 78: 141-4. PMid:9198144.

[3] Heit JA, Silverstein MD, Mohr DN, et al. Risk factors for deep vein thrombosis and pulmonary embolism: a population-based casecontrol study. Archives of Internal Medicine. 2000; 160: 809-15. PMid:10737280. https://doi.org/10.1001/archinte.160.6 .809

[4] Blom JW, Doggen CJ, Osanto S, et al. Malignancies, prothrombotic mutations and the risk of venous thrombosis. Jama. 2005; 293 : 715-22. PMid:15701913. https ://doi .org/10.1001/jama. 293 .6 .715

[5] Agnelli G, Bolis G, Capussotti L, et al. A clinical outcome-based prospective study on venous thromboembolism after cancer surgery: the @RISTOS project. Annals of Surgery. 2006; 243: 89-95. PMid:16371741. https ://doi.org/10.1097/01.sla.0000193 959.44677 .48

[6] Blom JW, Osanto S, Rosendaal FR. The risk of a venous thrombotic event in lung cancer patients: higher risk for adenocarcinoma than squamous cell carcinoma. Journal of Thrombosis and Haemostasis: JTH. 2004; 2: 1760-5. PMid:15456487. https: //doi.org/10.1111/j.1538-7836.2004.00928.x

[7] Blom JW, Osanto S, Rosendaal FR. High risk of venous thrombosis in patients with pancreatic cancer: a cohort study of 202 patients. European Journal of Cancer. 2006; 42: 410-4. PMid:16321518. https://doi.org/10.1016/j.ejca.2005.09.013

[8] Khorana AA, Francis CW, Culakova E, et al. Thromboembolism in hospitalized neutropenic cancer patients. Journal of Clinical Oncology: Official Journal of the American Society of Clinical Oncology. 2006; 24: 484-90. PMid:16421425. https ://doi.org/10.1200/ JC0. 2005.03 .8877

[9] Sallah S, Wan JY, Nguyen NP. Venous thrombosis in patients with solid tumors: determination of frequency and characteristics. Thrombosis and Haemostasis. 2002; 87: 575-9. PMid:12008937.

[10] Stein PD, Beemath A, Meyers FA, et al. Incidence of venous thromboembolism in patients hospitalized with cancer. The American Journal of Medicine. 2006; 119: 60-8. PMid:16431186. https: //doi.org/10.1016/j.amjmed.2005.06.058

[11] Trousseau A. Phlegmasia alba dolens. In: Trousseau A, editor. [Clinique Medicale de 1'Hotel Dieu de Paris 1865]. Paris: Balllier; 654712. French.

[12] Zwicker JI, Furie BC, Furie B. Cancer-associated thrombosis. Critical Reviews in Oncology/Hematology. 2007; 62: 126-36. PMid:17293122. https://doi.org/10.1016/j.critrevonc. 2 007.01 .001

[13] Rickles FR, Falanga A. Molecular basis for the relationship between thrombosis and cancer. Thrombosis Research. 2001; 102: V215-24. https://doi .org/10.1016/S0049-3848(01)00285-7

[14] Haddad TC, Greeno EW. Chemotherapy-induced thrombosis. Thrombosis Research. 2006; 118: 555-68. PMid:16388837. https://doi. org/10.1016/j.thromres. 2005.10.015

[15] Bick RL. Cancer-associated thrombosis. The New England Journal of Medicine. 2003; 349: 109-11. PMid:12853582. https: //doi.org/10.1056/NEJMp030086

[16] Otten HM, Mathijssen J, ten Cate H, et al. Symptomatic venous thromboembolism in cancer patients treated with chemotherapy: an underestimated phenomenon. Archives of Internal Medicine. 2004; 164: 190-4. PMid:14744843. https://doi.org/10.1001/arch inte.164.2.190

[17] Khorana AA, Francis CW, Culakova E, et al. Risk factors for chemotherapy-associated venous thromboembolism in a prospective observational study. Cancer. 2005; 104: 2822-9. PMid:16284987. https://doi.org/10.1002/cncr. 21496
[18] Khorana AA, Francis CW, Culakova E, et al. Thromboembolism is a leading cause of death in cancer patients receiving outpatient chemotherapy. Journal of Thrombosis and Haemostasis: JTH. 2007; 5: 632-4. PMid:17319909. https://doi.org/10.1111/j.1538 $-7836.2007 .02374 . \mathrm{x}$

[19] Falanga A. The incidence and risk of venous thromboembolism associated with cancer and nonsurgical cancer treatment. Cancer Investigation. 2009; 27: 105-15. PMid:19160098. https ://doi.or $\mathrm{g} / 10.1080 / 07357900802563028$

[20] Sorensen HT, Mellemkjaer L, Olsen JH, et al. Prognosis of cancers associated with venous thromboembolism. The New England Journal of Medicine. 2000; 343: 1846-50. PMid:11117976. https : //doi.org/10.1056/NEJM200012213432504

[21] Elting LS, Escalante CP, Cooksley C, et al. Outcomes and cost of deep venous thrombosis among patients with cancer. Archives of Internal Medicine. 2004; 164: 1653-61. PMid:15302635. https : //doi.org/10.1001/archinte.164.15.1653

[22] Heit JA, Mohr DN, Silverstein MD, et al. Predictors of recurrence after deep vein thrombosis and pulmonary embolism: A populationbased cohort study. Archives of Internal Medicine. 2000; 160: 761-8. PMid:10737275. https : //doi .org/10.1001/archinte.160.6 .761

[23] Debourdeau P, Kassab Chahmi D, Le Gal G, et al. 2008 SOR guidelines for the prevention and treatment of thrombosis associated with central venous catheters in patients with cancer: Report from the working group. Annals of Oncology: Official Journal of the European Society for Medical Oncology / ESMO. 2009; 20: 1459-71. PMid:19525362. https ://doi.org/10.1093/annonc/mdp052

[24] Geerts WH, Bergqvist D, Pineo GF, et al. Prevention of venous thromboembolism: American College of Chest Physicians EvidenceBased Clinical Practice Guidelines (8th Edition). Chest. 2008; 133: 381S-453S

[25] Lyman GH, Khorana AA, Falanga A, et al. American Society of Clinical Oncology guideline: recommendations for venous thromboembolism prophylaxis and treatment in patients with cancer. Journal of Clinical Oncology: Official Journal of the American Society of Clinical Oncology. 2007; 25: 5490-505. PMid:17968019. https://doi.org/10.1200/JC0.2007.14.1283

[26] Oremus M, Wolfson C, Perrault A, et al. Interrater reliability of the modified Jadad quality scale for systematic reviews of Alzheimer's disease drug trials. Dementia and Geriatric Cognitive Disorders. 2001; 12: 232-6. PMid:11244218. https://doi.org/10.1159/ 000051263

[27] Pelzer U, Opitz B, Deutschinoff G, et al. Efficacy of Prophylactic Low-Molecular Weight Heparin for Ambulatory Patients With Advanced Pancreatic Cancer: Outcomes From the CONKO-004 Trial. Journal of Clinical Oncology: Official Journal of the American Society of Clinical Oncology. 2015; 33: 2028-34. PMid:25987694. https://doi.org/10.1200/JC0.2014.55.1481

[28] Macbeth F, Noble S, Evans J, et al. Randomized Phase III Trial of Standard Therapy Plus Low Molecular Weight Heparin in Patients With Lung Cancer: FRAGMATIC Trial. Journal of Clinical Oncology: Official Journal of the American Society of Clinical Oncology. 2015. PMid:26700124.

[29] Lecumberri R, Lopez Vivanco G, Font A, et al. Adjuvant therapy with bemiparin in patients with limited-stage small cell lung cancer: Results from the ABEL study. Thrombosis Research. 2013; 132: 666-70. PMid:24491267. https://doi.org/10.1016/j.thromr es. 2013.09.026

[30] Lavau-Denes S, Lacroix P, Maubon A, et al. Prophylaxis of catheterrelated deep vein thrombosis in cancer patients with low-dose warfarin, low molecular weight heparin, or control: A randomized, con- 
trolled, phase III study. Cancer Chemotherapy and Pharmacology. 2013; 72: 65-73. PMid:23636449. https://doi.org/10.1007/ s00280-013-2169-y

[31] Zwicker JI, Liebman HA, Bauer KA, et al. Prediction and prevention of thromboembolic events with enoxaparin in cancer patients with elevated tissue factor-bearing microparticles: a randomized-controlled phase II trial (the Microtec study). British Journal of Haematology. 2013; 160: 530-7. PMid:23240761. https://doi.org/10.1111/ bjh. 12163

[32] Haas SK, Freund M, Heigener D, et al. Low-molecular-weight heparin versus placebo for the prevention of venous thromboembolism in metastatic breast cancer or stage III/IV lung cancer. Clinical and Applied Thrombosis/Hemostasis: Official Journal of the International Academy of Clinical and Applied Thrombosis/Hemostasis. 2012; 18: 159-65. PMid:22275397. https ://doi .org/10.1177/10760296 11433769

[33] Agnelli G, George DJ, Kakkar AK, et al. Semuloparin for thromboprophylaxis in patients receiving chemotherapy for cancer. The New England Journal of Medicine. 2012; 366: 601-9. PMid:22335737. https://doi.org/10.1056/NEJMoa1108898

[34] Levine MN, Gu C, Liebman HA, et al. A randomized phase II trial of apixaban for the prevention of thromboembolism in patients with metastatic cancer. Journal of Thrombosis and Haemostasis: JTH. 2012; 10: 807-14. PMid:22409262. https://doi .org/10.1111/ j.1538-7836.2012.04693.x

[35] Maraveyas A, Waters J, Roy R, et al. Gemcitabine versus gemcitabine plus dalteparin thromboprophylaxis in pancreatic cancer. European Journal of Cancer. 2012; 48: 1283-92. PMid:22100906. https://doi.org/10.1016/j.ejca.2011.10.017

[36] Tang BQ, Guo W, Yang RL, et al. Evaluation of efficacy and safety of rivaroxaban in the prevention of postoperative venous thromboembolism in adult patients with primary bone tumor undergoing knee operation. Zhonghua Yi Xue Za Zhi. 2012; 92: 2768-71. PMid:23290165.

[37] van Doormaal FF, Di Nisio M, Otten HM, et al. Randomized trial of the effect of the low molecular weight heparin nadroparin on survival in patients with cancer. Journal of Clinical Oncology: Official Journal of the American Society of Clinical Oncology. 2011; 29: 2071-6. PMid:21502549. https://doi.org/10.1200/JC0.2010.31.92 93

[38] Perry JR, Julian JA, Laperriere NJ, et al. PRODIGE: a randomized placebo-controlled trial of dalteparin low-molecular-weight heparin thromboprophylaxis in patients with newly diagnosed malignant glioma. Journal of Thrombosis and Haemostasis: JTH. 2010; 8: 1959-65. PMid:20598077. https://doi.org/10.1111/j.1538 $-7836.2010 .03973 . x$

[39] Young AM, Billingham LJ, Begum G, et al. Warfarin thromboprophylaxis in cancer patients with central venous catheters (WARP): An open-label randomised trial. Lancet. 2009; 373: 567-74. https : //doi.org/10.1016/S0140-6736(09)60205-1

[40] Agnelli G, Gussoni G, Bianchini C, et al. Nadroparin for the prevention of thromboembolic events in ambulatory patients with metastatic or locally advanced solid cancer receiving chemotherapy: a randomised, placebo-controlled, double-blind study. The Lancet Oncology. 2009; 10: 943-9. https://doi.org/10.1016/S1470-204 5 (09) $70232-3$

[41] Shukla PJ, Siddachari R, Ahire S, et al. Postoperative deep vein thrombosis in patients with colorectal cancer. Indian Journal of Gas- troenterology: Official Journal of the Indian Society of Gastroenterology. 2008; 27: 71-3. PMid:18695308.

[42] Sideras K, Schaefer PL, Okuno SH, et al. Low-molecular-weight heparin in patients with advanced cancer: a phase 3 clinical trial. Mayo Clinic Proceedings. 2006; 81: 758-67. PMid:16770976. https://doi.org/10.4065/81.6.758

[43] Klerk CP, Smorenburg SM, Otten HM, et al. The effect of low molecular weight heparin on survival in patients with advanced malignancy. Journal of Clinical Oncology: Official Journal of the American Society of Clinical Oncology. 2005; 23: 2130-5. PMid:15699479. https://doi.org/10.1200/JC0.2005.03.134

[44] Altinbas M, Coskun HS, Er O, et al. A randomized clinical trial of combination chemotherapy with and without low-molecularweight heparin in small cell lung cancer. Journal of Thrombosis and Haemostasis: JTH. 2004; 2: 1266-71. PMid:15304029. https://doi.org/10.1111/j.1538-7836.2004.00871.x

[45] Kakkar AK, Levine MN, Kadziola Z, et al. Low molecular weight heparin, therapy with dalteparin, and survival in advanced cancer: the fragmin advanced malignancy outcome study (FAMOUS). Journal of Clinical Oncology: Official Journal of the American Society of Clinical Oncology. 2004; 22: 1944-8. PMid:15143088. https://doi.org/10.1200/JC0.2004.10.002

[46] Levine M, Hirsh J, Gent M, et al. Double-blind randomised trial of a very-low-dose warfarin for prevention of thromboembolism in stage IV breast cancer. Lancet. 1994; 343: 886-9. https ://doi .org/10 .1016/S0140-6736 (94)90008-6

[47] Marassi A, Balzano G, Mari G, et al. Prevention of postoperative deep vein thrombosis in cancer patients. A randomized trial with low molecular weight heparin (CY 216). International Surgery. 1993; 78: 166-70. PMid:8394842.

[48] Rickles FR, Patierno S, Fernandez PM. Tissue factor, thrombin, and cancer. Chest. 2003; 124: 58S-68S. PMid:12970125. https: //doi.org/10.1378/chest.124.3_suppl.58S

[49] Prandoni P, Lensing AW, Buller HR, et al. Comparison of subcutaneous low-molecular-weight heparin with intravenous standard heparin in proximal deep-vein thrombosis. Lancet. 1992; 339: 441-5. https://doi.org/10.1016/0140-6736(92)91054-C

[50] Amirkhosravi A, Mousa SA, Amaya M, et al. Assessment of antimetastatic effects of anticoagulant and antiplatelet agents using animal models of experimental lung metastasis. Methods in Molecular Biology. 2010; 663: 241-59. PMid:20617422. https://doi.org/ 10.1007/978-1-60761-803-4_10

[51] Borsig L. Antimetastatic activities of heparins and modified heparins. Experimental evidence. Thrombosis Research. 2010; 125 Suppl 2: S66-71. https://doi.org/10.1016/S0049-3848(10 ) 70017-7

[52] Lecumberri R, Paramo JA, Rocha E. Anticoagulant treatment and survival in cancer patients. The evidence from clinical studies. Haematologica. 2005; 90: 1258-66. PMid:16154849.

[53] Ahrens I, Lip GY, Peter K. New oral anticoagulant drugs in cardiovascular disease. Thrombosis and Haemostasis. 2010; 104: 49-60. PMid:20539909. https://doi .org/10.1160/TH09-05-0327

[54] van der Hulle T, den Exter PL, Kooiman J, et al. Meta-analysis of the efficacy and safety of new oral anticoagulants in patients with cancer-associated acute venous thromboembolism. Journal of Thrombosis and Haemostasis: JTH. 2014; 12: 1116-20. PMid:24819040. https://doi.org/10.1111/jth.12605 